\title{
Belgeo
}

Revue belge de géographie

\section{Institutionalisation de la géographie et rapports sociaux de genre : stratégies féminines et masculines dans l'université espagnole}

Institutionalisation of geography and gender relations : strategies of women and men in Spanish universities

\section{Maria Dolors Garcia Ramon, Anna Ortiz et Herminia Pujol}

\section{OpenEdition}

\section{Journals}

Édition électronique

URL : http://journals.openedition.org/belgeo/6251

DOI : 10.4000/belgeo.6251

ISSN : 2294-9135

Éditeur :

National Committee of Geography of Belgium, Société Royale Belge de Géographie

\section{Référence électronique}

Maria Dolors Garcia Ramon, Anna Ortiz et Herminia Pujol, « Institutionalisation de la géographie et rapports sociaux de genre : stratégies féminines et masculines dans l'université espagnole », Belgeo [En ligne], 1-2 | 2012, mis en ligne le 15 décembre 2012, consulté le 21 avril 2019. URL : http:// journals.openedition.org/belgeo/6251; DOI : 10.4000/belgeo.6251

Ce document a été généré automatiquement le 21 avril 2019

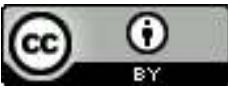

Belgeo est mis à disposition selon les termes de la licence Creative Commons Attribution 4.0 International. 


\section{Institutionalisation de la géographie et rapports sociaux de genre : stratégies féminines et masculines dans l'université espagnole ${ }^{1}$}

Institutionalisation of geography and gender relations : strategies of women and men in Spanish universities

Maria Dolors Garcia Ramon, Anna Ortiz et Herminia Pujol

\section{Introduction}

1 L'objectif de cet article est d'étudier les carrières professionnelles à l'intérieur des départements de géographie en Espagne, espaces professionnels qui deviennent de plus en plus compétitifs et masculins. En Espagne, comme dans de nombreux pays occidentaux, des processus de néolibéralisation s'installent à l'intérieur des universités (Dowling, 2008). Ceux-ci incluent la diffusion de logiques compétitives et de marché, la montée en puissance des procédures d'audits et de requis de responsabilité/imputabilité ainsi que le remplacement de fonds publics par des fonds privés. Ces processus ont un impact important sur les perceptions et pratiques relatives à la carrière professionnelle des géographes espagnols. Dans ce contexte de transformation, le nombre relatif de femmes géographes décroît et leur statut professionnel ne s'améliore pas.

Nous nous concentrerons sur les stratégies et pratiques de ces femmes géographes - ainsi que sur leurs sentiments et expériences - quant à la conciliation vie professionnelle/vie familiale, l'obtention de promotions et l'accès au pouvoir décisionnel dans le monde académique. Notre recherche comprend également un état des lieux de la situation des hommes dans le monde académique pour pouvoir comparer les carrières académiques "masculines" et "féminines". L'article se base principalement sur l'analyse qualitative (interviews en profondeur). De nombreuses études ont été publiées à ce sujet en 
géographie (de Zelinsky, 1973a, 1973b ; McDowell, 1979; Momsen, 1980 ; Crang, 2003 et Creton, 2007), mais peu d'études qualitatives ont été réalisées et nous estimons que ce type d'analyse représente la meilleure méthode pour analyser cette question. La raison pour laquelle nous nous focalisons sur la géographie tient au fait qu'en tant que géographes nous sommes familières du contexte, familiarité qui constitue un requis indispensable pour l'interprétation des résultats d'une étude qualitative. Par ailleurs, la géographie se situant entre les sciences sociales et les sciences physiques, les résultats de notre recherche pourront s'appliquer à un bon nombre de disciplines scientifiques.

En 1988 nous publiions un rapport sur la situation des femmes géographes dans le monde académique espagnol (Garcia-Ramon et al., 1988) et nous avions découvert que leur présence était relativement importante (autour d'un tiers du corps professoral, bien que concentré dans des positions subalternes), surtout si comparée à la proportion des femmes dans la géographie anglo-saxonne (Monk, 1994). Or, actuellement cette appréciation changerait vu que la proportion de femmes dans le corps professoral des géographes a diminué (Pujol, 2004). Certaines études récentes indiquent que dans les universités catalanes le ratio entre hommes et femmes dans les équipes professorales en géographie est passé de 1,6 hommes pour une femme en 1996 (Lluch et al., 1998) à 2,1 hommes pour une femme en 2008 (Pujol et al., 2008; Pujol, 2011). Cette diminution de la présence féminine est probablement due au fait que dans les années 80 l'image de la géographie en Espagne était associée à la pratique professionnelle des femmes enseignantes de lycée et non à une image plus masculine - comme de nos jours. Cette masculinisation nous semble liée à la récente croissance de la géographie technique et à la profession de géographe en tant que tel (Garcia-Ramon \& Pujol, 2004 ; Pujol, 2011).

\section{Méthodologie du travail de terrain}

Le travail de terrain de cette recherche a été réalisé entre 2007 et 2009 (Pujol, 2011). Il comprend les interviews en profondeur d'un total de 57 personnes appartenant au corps professoral de 12 universités publiques espagnoles (aux alentours d'un quart du total du corps enseignant en géographie de ces universités). De cet échantillon, 43 interviewés sont des femmes et 14 des hommes. Tous sont nommés en tant que profesor titular (ce qui correspond plus ou moins au poste de maître de conférences) ou catedrático (statut qui correspond à celui de professeur des universités), les deux principales catégories professionnelles présentes dans les universités espagnoles. Les principaux critères d'inclusion dans l'échantillon étaient l'âge, la situation familiale et le statut professionnel à l'intérieur du monde académique.

5 Le groupe d'âge le plus représenté dans notre échantillon est celui des 46-60 ans. Les principales caractéristiques, de notre échantillon, utiles pour la présente étude sont : (i) la majorité des femmes d'âge moyen ont un conjoint et les femmes célibataires sont soit catedráticas, soit de très jeunes profesor titular, (ii) tous les hommes ont une conjointe et tous, sauf un, ont des enfants, (iii) près d'un tiers des femmes n'ont pas d'enfants. Et il faut dire que 11 des 13 femmes catedráticas ont vécu un divorce alors qu'aucun de leur collègues masculins de même niveau professionnel n'a vécu une telle rupture.

6 Chaque interview a duré environs 90 minutes, a été enregistrée et retranscrite et le discours de chaque interview a été analysé au moyen de codes ou de mots clés. L'interview couvrait un large éventail de thèmes mais l'étude présentée ne reprend que trois d'entre eux: (i) l'équilibre travail-vie privée, (ii) le processus de promotion 
académique et la question du pouvoir décisionnel et (iii) les fonctions d'encadrement et les postes à responsabilités institutionnelles.

\section{Productivité scientifique et conciliation travail-famille}

7 Quant à l'équilibre entre vie familiale et travail académique, la productivité scientifique et les différences de genre émergent comme des éléments clés. La plus faible productivité des femmes dans le monde académique a été expliquée par les difficultés à concilier vie professionnelle et vie familiale ainsi que par l'accès plus restreint des femmes aux réseaux informels et aux contacts personnels qui facilitent la publication. Les études de Garcia Ramon et Castañer (1992) et de Ramiro (2005) relèvent l'importance du rôle familial des femmes quand on analyse leur production. Toutefois, ces études rappellent également que la plus faible proportion des femmes dans le corps enseignant des départements universitaires de géographie représente une explication en elle-même du moindre nombre de publications de ces femmes.

8 Toutefois, certains auteurs ne partagent que partiellement ces explication et Winkler (2000) observe que les femmes célibataires publient, dans certains cas, également en dessous de la moyenne. Quand on les interroge sur ce fait, leur réponse évoque toujours le sentiment d'exclusion et d'isolement social et intellectuel qui entraîne un accès limité aux ressources sociales. Ce manque d'intégration dans les réseaux sociaux des départements mène à une exclusion de fait des négociations invisibles entre les membres de ces départements et éventuellement débouche sur une moindre capacité à identifier de possibles collaborateurs ou même des assistants de recherche. Acquérir de l'influence dans des groupes informels requiert du temps libre considérable et c'est exactement ce que de nombreuses femmes, et spécialement les femmes mariées avec des enfants, ne peuvent s'offrir. Des conclusions similaires ont été établies en ce qui concerne les carrières académiques des femmes dans les universités espagnoles. Les promotions sont conditionnées à l'obtention d'un statut basé sur la production scientifique qui dépend des groupes informels dans lesquels les femmes sont marginales. Publier des études requiert une légitimité souvent établie par des groupes informels où les femmes ne sont pas visibles (Garcia de León et al., 2001).

9 Certains auteurs (Deem, 2003; Wood \& Newton, 2006) ont analysé les carrières universitaires d'hommes et de femmes dans le but de découvrir dans quelle mesure ces carrières et les attentes qui leur sont liées ont été influencées par les relations de genre. Les femmes ressentent que leurs obligations familiales ont entravé ou retardé leur carrière et elles perçoivent un parti pris sexiste dans les décisions relatives aux promotions. Les hommes, pour leur part, ne perçoivent pas la paternité comme un problème et reconnaissent que s'ils avaient été femmes, il aurait été probablement plus difficile pour eux d'atteindre leur position académique actuelle. Ils ne mentionnent pas dans la même mesure que les femmes le sentiment d'incompatibilité entre devoirs familiaux et carrière académique.

10 Une étude sur le professorat féminin dans les universités publiques espagnoles (Carreño et al., 1998) a mis en évidence que de nombreuses femmes se plaignent de leur isolement à l'intérieur des départements universitaires et qu'elles dénoncent des conflits hiérarchiques basés sur le genre ainsi qu'un paternalisme masculin marqué. Elles relèvent qu'elles doivent faire d'importants sacrifices dans leur vie privée. Elles mentionnent 
également que la difficulté de concilier vie familiale et travail est souvent une source d'insatisfaction et de manque d'auto-estime.

Dans notre échantillon, la majorité des interviewés (femmes et hommes) s'accordent sur le fait que les femmes font face à un double fardeau : leur carrière professionnelle et une plus grande part de responsabilité dans l'organisation de la vie domestique. Parallèlement, toutes les femmes interrogées soulignent que l'horaire flexible de travail est l'aspect le plus apprécié de la vie universitaire en ce qui concerne la combinaison travail/vie familiale, particulièrement si elles ont des enfants. Enseigner dans les auditoires et assurer un encadrement personnalisé aux étudiants requièrent des horaires rigides alors que les tâches administratives et la recherche peuvent s'adapter dans une certaine mesure à leur besoins et horaires (Tobio, 2005 ; Álvarez Valverde, 2006 ; Fundació Maria Aurèlia Campmany, 2007). Assez curieusement, la plupart des hommes n'abordent pas la flexibilité horaire dans leurs interviews.

"Je pense que nous sommes privilégiées en comparaison d'autres femmes, celles qui travaillent dans un magasin, dans une usine, un hôpital... Nous ne devrions pas nous poser en victimes. Les femmes qui travaillent avec des horaires rigides et pour qui la conciliation est très difficile, et qui plus est, dont le travail est moins gratifiant... Je veux dire, tous nos conflits sur comment partager notre temps ne sont rien comparés avec le vrai monde, le monde en dehors du monde académique" (Clara, 31-45 ans, mariée avec des enfants, profesora titular).

12 Un horaire de travail flexible est considéré comme avantageux non seulement par les femmes avec des enfants en bas âge mais aussi par celles qui s'occupent de parents dépendants, particulièrement des parents âgés. Néanmoins, de nombreuses femmes mentionnent le "piège" de la flexibilité car, selon l'une d'entre elles, il faut compenser "les heures volées dédiées à la famille".

Malgré les avantages perçus des horaires flexibles de travail, l'idée de sacrifice est une constante dans le discours des femmes interrogées, indépendamment de leur âge. Cette idée est particulièrement liée aux années durant lesquelles elles ont, ou ont eu, des enfants en bas d'âge, années durant lesquelles elles ont dû récupérer les heures consacrées à leurs enfants pendant le weekend ou tard la nuit. Fréquemment cette situation engendre du stress.

"Je devais travailler tard la nuit, ce qui a fortement conditionné mes heures de sommeil... Le paradigme de la femme au foyer tu l'as en permanence à l'esprit, et quand as-tu du temps ?... tard la nuit" (Olga, plus de 61 ans, mariée et avec enfants, profesora titular).

14 Se sentir "coupable" de ne pas s'occuper suffisamment des enfants ou même des parents âgés est un des sentiments des plus communs parmi les femmes ayant des obligations familiales. Par exemple, une femme comparant ses responsabilités avec celles de son frère raconte :

"Son épouse le remplaçait (en s'occupant de la mère de celui-ci)... il n'avait jamais à se sentir coupable, jamais... jamais... et nous (les femmes) nous devons ressentir ce sentiment... nous avons ce fardeau (la culpabilité). Comme 'mauvaise' mère... et ensuite comme 'mauvaise' fille... malgré tout tu dois prendre des décisions..." (Sara, plus de 61 ans, mariée avec des enfants, catedrática).

15 Ce sentiment de culpabilité n'est jamais mentionné par les hommes.

16 Dans la plupart des cas, le recours à la famille - spécialement aux grands-mères (la ainsi nommée "solidarité féminine intergénérationnelle", selon Altaba, 2004) - ou le recours 
aux services payants sont les stratégies que les femmes emploient pour faire face à ce double fardeau.

Une étude de Deem (2003) sur les hommes et les femmes dans différentes universités britanniques a mis à jour que les femmes ressentaient que leurs responsabilités familiales avaient ralenti leur carrière alors que les hommes généralement ne considéraient pas que la paternité avait représenté un problème pour eux. Ils admettent, par contre, que s'ils avaient été femmes, ils auraient probablement rencontré plus de difficultés pour atteindre leur statut professionnel actuel. Dans notre échantillon, les femmes mentionnent également que leurs responsabilités familiales peuvent avoir ralenti leur carrière, bien qu'elles ne le disent pas ouvertement. Les hommes, à quelques exceptions près, ne mentionnent pas ce point.

Il vaut la peine de mentionner que certains hommes décrivent la vie familiale comme un facteur stabilisant très important pour leur bien-être émotionnel ainsi que pour leur carrière. Par exemple :

"Je pourrais mentionner certains coûts (relatifs à la famille) mais je devrais plutôt mentionner que la famille a des effets compensatoires, et même stimulants... ainsi j'ai un soutien (pour ma carrière), et je pense que c'est très important d'avoir quelqu'un avec qui partager ses difficultés" (Eudalt, entre 46 et 60 ans, avec conjoint et deux enfants, professor titular).

19 Assez curieusement ce facteur de stabilité émotionnelle lié à la famille n'est jamais exprimé dans les interviews des femmes, bien que certaines d'entre elles ressentent probablement des sentiments similaires.

Les femmes sans enfants sont pleinement conscientes des difficultés qu'elles auraient rencontrées, si elles avaient eu des enfants, pour mener à bien leurs activités académiques (par exemple la participation à des congrès, la réalisation de travail de terrain de longue haleine, etc.). Ces femmes parfois pensent que les enfants représentent un handicap pour la carrière académique.

“Ce n'est pas un hasard si je n'ai pas d'enfants. La raison n'est pas biologique, elle est sociologique. Je n'ai pas eu le temps, je devais dédier tous mes efforts à ma carrière" (Gala, entre 46 et 60 ans, avec un conjoint et sans enfants, profesora titular). "J'ai consacré toute ma vie à ma carrière académique... Je n'ai jamais ressenti la nécessité d'être maman. Et cela ne me manque pas maintenant car je perçois clairement que les deux choses sont incompatibles... J'ai eu une vie avec une disponibilité totale, sans restrictions d'horaires, si tu dois voyager tu n'a qu'à partir, si tu as besoin de plus d'heures de travail, tu les prends... telle a été ma vie" (Mila, entre 46 et 60 ans, divorcée et sans enfants, catedrática).

21 Les difficultés qu'elles prévoient quant à la conciliation vie familiale/carrière professionnelle (devenue progressivement plus compétitive depuis les années 90) sont perçues par les femmes, selon la majorité de nos interviewées, dès le début de leur carrière. C'est une tendance observable dans nos universités que, parmi les doctorants bénéficiant d'une bourse, un nombre significativement plus élevé d'étudiantes abandonnent leur thèse avant l'obtention de leur doctorat. Une explication plausible est que la carrière académique est trop exigeante pour une jeune femme qui ne veut pas renoncer à fonder une famille et avoir des enfants.

"Le drame c'est qu'elles abandonnent quand elles sont doctorantes avec une bourse. Les doctorants continuent et les doctorantes abandonnent au profit d'un emploi stable quelconque. La vie académique est trop exigeante, la recherche est trop compétitive, trop longue... elle est perçue comme plus incertaine qu'elle ne l'est en réalité... Après la thèse, tu as le processus de titularisation, toujours incertain, que 
va-t-il m'arriver ?... Elles n'apprécient point cette situation car elles veulent avoir des enfants, elles veulent rembourser le prêt hypothécaire... ce n'est pas qu'elles ne veulent pas travailler mais elles veulent le faire de 9 à 17 heures ou de 10 à 19 heures de manière à savoir exactement ce qu'on attend d'elles..." (Tania, plus de 61 ans, divorcée avec enfants, catedrática).

\section{Le processus de promotion et d'acquisition de pouvoir} académiques sont fortement "genrisées" vu que peu de femmes atteignent le statut académique le plus élevé, celui de catedrática. Il y a un obstacle qui se présente comme insurmontable pour les femmes mais pas pour les hommes. On peut supposer que la question de la promotion et du pouvoir est liée à un héritage historique car les femmes ont commencé à intégrer le corps professoral plus récemment que les hommes. Néanmoins, Cortazar et García de Leon (1997) ainsi que Simelio et Rovetto (2008) ont défendu l'idée que les statistiques espagnoles montrent que les promotions successives d'étudiantes et de femmes professeurs avec les qualifications requises pour être promues au statut de catedrática ont été suffisantes. Ils établissent également que le pourcentage $\mathrm{du}$ professorat féminin est anormalement bas et que ceci est le fruit des cercles de pouvoir androcentriques à l'intérieur du monde universitaire : "l'effet du club des vieux copains" (la cooptation, par les hommes, presque toujours inconsciente de candidats parmi "leurs égaux"). Pour remédier à cette situation, Miyares (2008) défend l'introduction de quotas universitaires comme une mesure politique pour une université plus égalitaire, dans laquelle les femmes auraient un poids plus important dans les prises de décisions.

Brooks (1997) souligne que dans les universités britanniques les femmes ont souvent de plus lourdes charges d'enseignement, d'administration ainsi qu'un plus grand nombre d'heures consacrées à l'encadrement que les hommes. Les femmes participent fréquemment à des comités requérant beaucoup de temps (admission d'étudiants, etc.) mais sont rarement présentes dans les instances décisionnelles les plus importantes ou des postes de direction. Cette situation n'aide, en rien, leur carrière académique et leur promotion vu qu'elles ont moins de temps pour la recherche et la publication, véritables indicateurs de la productivité académique.

Le système d'évaluation qui prévaut dans le monde académique, basé sur des critères qui ignorent les spécificités des carrières professionnelles féminines est considéré par les femmes comme sous-évaluant ou marginalisant leurs contributions. Il a fait l'objet de nombreuses critiques (Martinez, 2008; Wilson \& Nutley, 2003). Une carrière académique standard est structurée selon la perception masculine du succès et le système méritocratique adopté reflète et reproduit les pratiques discursives masculines tout en discriminant la majorité des femmes et certains hommes (Knight \& Richards, 2003).

L'image de la carrière académique est entièrement masculine, suivant l'image traditionnelle du scientifique: un être qui vit dans une "tour d'ivoire" entièrement consacré à la science et non distrait par d'autres préoccupations triviales comme celles relatives à la vie familiale. Cette image a des répercussions sur tous les processus d'évaluation qui mènent aux postes académiques et qui valorisent non seulement l'intensité de la production scientifique mais également le caractère ininterrompu de celle-ci (Benshop \& Bround, 2003 ; Wilson \& Nutley, 2003). 
26 Toutes les femmes de notre échantillon sont pleinement conscientes du fait que les normes d'évaluation du travail académique découlent de ce modèle ou de cette image : une image franchement masculine. Ce sentiment est fortement exprimé par les femmes avec des enfants et celles qui ont plus de 45 ans et qui n'ont pas atteint le statut de catedrática. Les membres de ces deux groupes ressentent qu'elles ont dans la vie des intérêts plus larges et variés que les hommes. Pour cela elles disent qu'elles ne veulent pas s'engager dans la féroce compétition généralement associée à l'obtention d'une chaire (ou peut-être est-il trop tard pour elles de se lancer dans cette compétition).

Pour Homer, par contre, la promotion de profesor titular à catedrático n'était pas uniquement une question d'inertie ou la suite logique de sa carrière. Elle représentait également une manière de répondre aux attentes de son environnement professionnel. En effet, il raconte :

“On s'attendait à ce que je me présente au poste de catedrático. Qu'auraient pensé mes collègues du département si je n'avais pas tenté le coup ?... C'était considéré comme une évolution normale si je voulais continuer à diriger un groupe de recherche, obtenir des fonds, publier... alors je devais devenir catedrático" (Homer, entre 46 et 60 ans, marié avec enfants, catedrático).

$28 \mathrm{Au}$ contraire, les expériences vécues par les femmes lors de l'obtention d'une chaire semblent avoir été plus compliquées. Comme Bernat le reconnaît lui-même, "les femmes qui veulent devenir catedráticas ont toujours été stigmatisées comme très ambitieuses". En guise de conclusion, l'obtention d'une chaire est considérée pour les hommes comme le résultat naturel d'une brillante carrière académique, tandis que pour les femmes une telle nomination est perçue comme le résultat d'une ambition "contre nature".

En ce qui concerne la promotion, il est évident que les hommes ont trouvé plus facilement de l'aide dans les moments stressants de leur carrière académique : par exemple durant la rédaction de leur thèse ou pendant la préparation des concours publics. En particulier, ils ont fréquemment reçu l'aide de leur épouse. Une femme professeur raconte :

"Je pense que tout a été bien plus facile pour eux [les hommes]. Ils avaient le support de leur famille. Principalement l'épouse... Je ne veux pas dire qu'ils ne font rien... mais tout est plus simple pour eux. Vois-tu, [dans mon département] il y a six hommes catedráticos et seulement une femme avec le même titre" (Neus, entre 46 et 60 ans, divorcée et maintenant avec un conjoint, avec enfants, catedrática).

Un catedrático du même département semble confirmer cette situation quand il explique qu'il a toujours eu de l'aide de la part de son épouse - qui n'exerce pas de profession en dehors du foyer - et pas uniquement pour les tâches domestiques et familiales mais également pour des tâches professionnelles. Si nécessaire, la famille élargie prenait soin des enfants pour permettre aux parents de ceux-ci de répondre aux exigences académiques de l'époux dans des moments tendus.

“Elena [m'aidait] en introduisant les statistiques les plus élémentaires et en réunissant les données... disons qu'elle m'a aidé dans toute sorte de tâches 'administratives' lorsque j'en avais besoin. Et si le travail était envahissant, alors les enfants étaient envoyés chez les sœurs d'Elena pour qu'elles s'en occupent durant un moment. Les enfants adoraient cela!" (Roger, plus de 61 ans, marié avec des enfants, catedrático).

31 Certains hommes mentionnent que l'effort fourni pour leur propre promotion peut avoir freiné la carrière de leur partenaire mais ils semblent également ne point se sentir coupables de cela. 
“Elle se plaint continuellement que moi j'ai fait carrière alors qu'elle en est toujours au même point que lorsque nous avons commencé... que s'est-il passé ?... et elle me tuerait si elle m'entendait, c'est que je ne suis pas sûr que ce soit de ma faute, c'est plutôt qu'elle n'a pas voulu faire carrière" (Cosme, moins de 45 ans, avec partenaire et deux enfants, profesor titular).

académique semble émerger. Un modèle qui contredit l'image de l'universitaire vivant dans une "tour d'ivoire" et entièrement dédié à la science sans être distrait par des préoccupations "triviales" comme celles découlant de la vie familiale.

\section{Service : les postes de responsabilité institutionnelle}

Tomas et al. (2008) suggèrent que malgré la participation croissante des femmes dans les instances dirigeantes des universités espagnoles, celles-ci restent moins visibles du fait de leur style d'intervention moins agressif. Elles utilisent des expressions comme "à mon avis", "de mon point de vue", contrairement au style masculin. Elles recherchent plus le dialogue et le consensus et s'intéressent plus au processus décisionnel qu'à la décision en elle-même. Il existe également une spécificité sociodémographique propre à ces femmes qui occupent des fonctions administratives : la plupart d'entre elles sont plus âgées que leurs collègues masculins vu que généralement ces femmes ont des enfants déjà âgés. 
38 En Angleterre, on a observé une présence croissante des femmes dans des fonctions administratives (Brooks, 1997). Fonctions que les hommes évitent car de tels postes empiètent sur le temps dédié à la recherche et sont généralement considérés comme des fonctions d'encadrement et donc typiquement féminines. Voilà pourquoi Hanson (2007) conseille aux femmes géographes d'éviter de tels postes au début de leur carrière afin de pouvoir disposer de plus de temps pour la recherche et l'enseignement.

À cet égard, il est important de rappeler que depuis les années 80, la plupart des fonctions à responsabilité et de décision, dans les universités espagnoles, sont soumises au vote d'un large corps électoral (comprenant également le personnel non permanent et les représentants des étudiants) et que ces charges électives de direction partagent la prise de décision avec des comités également élus. En fait, cette structure permet uniquement une faible marge de pouvoir personnel (et de responsabilité). C'est dans ce contexte qu'un nombre croissant de femmes ont atteint de tels postes dans leur département ou faculté. Une femme de notre échantillon nous a raconté :

"En général mes collègues masculins sont réticents à prendre des responsabilités ; ils ne le font que lorsqu'ils n'ont pas le choix. Les responsabilités administratives à l'université sont uniquement une source de travail et de maux de tête et le bonus salarial n'en vaut pas la chandelle... Je ne suis pas catedrática... Je n'ai guère publié parce que j'ai passé de nombreuses heures à des tâches administratives... J'y ai consacré mon temps et je pense que je suis douée pour ces tâches. Je me dis 'travaille pour le bien collectif' parce que cela prend de nombreuses heures et que peu de personnes sont prêtes à s'y consacrer. Tu reçois peu d'argent et peu de reconnaissance" (Ginesta, entre 46 et 60 ans, mariée et avec des enfants, profesor titular).

40 Les femmes interviewées qui avaient atteint un poste à responsabilité à l'université (comme vice-recteur, doyenne, directrice de département ou d'un centre de recherche) étaient toutes célibataires, en couple mais sans enfants, ou encore, étaient soit mariées ou divorcées avec des enfants déjà âgés. Une des femmes qui occupait un de ces postes (doyenne de faculté) a expliqué que, quand elle a dû former son équipe de travail, le monde universitaire commençait à appliquer les récentes normes espagnoles sur l'équité de genre dans tous les comités et instances de direction de l'université. Elle se heurta à de nombreux problèmes au moment d'inviter ses collègues à participer à une équipe équilibrée en termes de genre. Assez curieusement, elle reçut des réponses plutôt différentes des hommes et des femmes occupant des postes universitaires similaires ( profesor titular) et ayant le même âge (la quarantaine). Les hommes qui refusèrent, s'excusèrent en mentionnant leur carrière professionnelle et la nécessité de dédier plus de temps à la recherche; les femmes par contre, expliquèrent que ce n'était pas le moment adéquat pour elles, car elles avaient des enfants en bas âge ou des parents âgés dont elles avaient la charge.

41 Il semble que notre recherche empirique confirme certaines analyses avancées dans la littérature scientifique sur les carrières féminines et masculines. Selon Perez Sedeño et al. (2008), le statut et la reconnaissance sociale associés aux professions généralement masculines sont intrinsèquement liés aux postes de responsabilité et de pouvoir qui requièrent une bonne dose d'ambition et un intense dévouement. Ces auteurs considèrent que la "culture des longues heures" et d'absolu dévouement au travail ainsi que l'ambition et la compétitivité ne sont pas "des valeurs intrinsèquement féminines" vu que l'on suggère aux femmes de donner priorité aux valeurs interpersonnelles et affectives et 
non à leurs objectifs professionnels (Garcia de Cortázar \& García de León, 1997 ; García de León \& Alonso, 2001).

Dans un milieu universitaire toujours plus compétitif, ce n'est pas une surprise si notre enquête de terrain a mis en évidence un manque général d'intérêt des deux parts (femmes et hommes) pour occuper des postes de responsabilité administrative, vu que ces postes privent les professeurs de temps pour la recherche et précarisent l'évaluation des $\mathrm{CV}$ basé sur les publications. Néanmoins, dans le cas de femmes, nous observons que certaines d'entre elles acceptent de telles responsabilités quand elles sont déjà nommées, qu'elles ont déjà des enfants âgés et qu'elles se perçoivent comme n'ayant pas de réelles chances de devenir catedráticas.

La perception que les femmes doivent prouver leur aptitude et valeur de manière plus appuyée que les hommes apparaît dans plusieurs interviews, en particulier dans le cas des femmes occupant des fonctions administratives ou ayant des postes à responsabilité. Il y a une nécessité constante pour ces femmes de prouver qu'elles sont suffisamment compétentes pour se conformer aux règles du jeu masculin (Krefting, 2003). Cette situation est présente dans le domaine de la recherche mais également lors des réunions où - certaines femmes nous l'ont raconté - elles se sentaient d'une certaine manière “invisibles" pour leurs collègues masculins et qu'elles devaient donner une image plus dure que celle qu'elles auraient bien voulu projeter.

"Tu dois être sûre de toi, sans hésitations... Je devais projeter une image bien plus dure que celle que j'aurais voulu donner... tu vois que tes arguments ne sont pas pris en compte. Parfois tu as émis un avis et tes collègues masculins l'oublient... ils se souviennent du contenu mais pas de qui a avancé l'idée... alors, qu'entre eux, ils se reconnaissent parfaitement. Les réflexions présentées par des femmes sont d'une certaine manière l'apport de gouttes dans l'océan" (Mila, entre 46 et 60 ans, divorcée et sans enfants, catedrática).

\section{Considérations finales}

$44 \mathrm{Au}$ cours de la dernière décennie les carrières académiques dans les universités espagnoles sont devenues plus longues et plus rudes. Une représentation qui se veut en relation avec la notion de compétitivité (mais aussi avec la masculinité) influence tous les processus d'évaluation qui mènent aux divers statuts universitaires. Les femmes trouvent donc plus difficilement leur chemin dans le monde académique (ainsi que l'accès aux promotions), principalement à cause de leurs responsabilités familiales.

Néanmoins, il est certain que les horaires de travail flexibles sont perçus par les femmes comme un grand avantage permettant de concilier travail et vie familiale. Il est également clair que la majorité des femmes interrogées ont atteint un bon équilibre (acceptable pour elles) entre la vie familiale et la vie professionnelle, même si nombre d'entre elles abandonnent la possibilité d'être promues au plus haut niveau académique (titulaire d'une chaire). Ce n'est pas dit très ouvertement, mais être catedrático est considéré pour les hommes comme le résultat "naturel" d'une brillante carrière universitaire, alors que pour les femmes l'obtention d'un tel statut est considérée comme le résultat d'une ambition "contre nature".

Excepté certains cas, où les tâches ménagères et familiales sont réellement partagées par les deux conjoints, les femmes universitaires se chargent d'une majeure partie de celles-ci dans leur vie de tous les jours. Le sentiment général est que les tâches familiales sont un 
obstacle à la carrière universitaire des femmes, bien que la prise en charge par ces femmes des principales responsabilités familiales génère également une certaine satisfaction personnelle pour celles-ci. En revanche certains hommes évoquent la famille comme un élément stabilisant. Les obligations familiales sont également le principal frein à l'acceptation de responsabilités institutionnelles dans les postes de direction.

Les femmes catedráticas que nous avons interviewées laissent entendre qu'elles ont dû suivre le soi-disant "modèle masculin" de la carrière universitaire, c'est-à-dire qu'elles ont dû donner la priorité à leur carrière professionnelle en payant un prix élevé dans leur vie personnelle et académique (ce n'est pas un hasard si 10 des 12 femmes catedráticas sont divorcées ou célibataires et qu'aucun des 9 hommes avec le même statut ne se retrouvent dans la même situation).

Néanmoins il y a des signes positifs vers un changement dans le futur. Dans le discours des interviews, de nombreuses femmes ainsi que d'un petit nombre d'hommes jeunes tentent de construire un modèle de carrière académique plus équilibré en ce qui concerne le genre (et il est évident qu'ils recherchent un tel équilibre dans leur vie de tous les jours). Ce nouveau modèle (qui se crée à travers leurs stratégies, comprenant de la résistance envers le modèle actuel mais aussi des efforts considérables pour créer un nouveau modèle) a été dénommé dans la littérature féministe "modèle hybride ou modèle métissé", un modèle qui s'imbrique quelque part entre le modèle masculin traditionnel et le modèle féminin (García de Cortazar, 1997). Ce changement, néanmoins, sera difficile, demandera du temps et des efforts, ainsi que des politiques publiques efficaces contre la discrimination, non seulement en ce qui concerne la vie familiale, mais aussi des mesures spécifiques au monde universitaire.

\section{BIBLIOGRAPHIE}

ALTABA M. S. (2004), Conciliación de la vida laboral y familiar. El quiero y no puedo de la madre trabajadora. Familia y Educación, AGEA (Asociación de grupos de Estudio de Actualidad), http:// agea.org.es (15/12/2007).

ÁLVAREZ VALVERDE, T. (2006), La conciliación de la vida personal, laboral y familiar ¿una utopía la corresponsabilidad? Codo con codo : comparte tareas y responsabilidades domésticas, Granada, Ayuntamiento de Granada.

BENSCHOP Y. \& BROUNS M. (2003), “Crumbling ivory towers : academic organizing and its gender effects", Gender, Work and Organization, 10, 2, pp. 194-212.

BROOKS A. (1997), Academic Women, The society for Research into Higher Education, Bristol (USA), Open University Press and SRHE.

CARREÑO A., FABRA M.L., LLORET C., PALLEJÀ M., PASTOR C. \& PÉREZ DE LARA N. (1998), La carrera de les professores universitàries, Bellaterra, Institut de Ciències de l'Educació de la Universitat Autònoma de Barcelona.

CRANG M. (2003), "Malestream geography : gender patterns among UK geography faculty", Environnement and Planning A, 35, pp. 1711-1716. 
CRETON D. (2007), “Gender issues in French Geography”, Belgeo, 8, 3, pp. 313-322.

DEEM R. (2003), "Gender, organizational cultures and the practices of manager-academics in UK Universities”, Gender, Work and Organization, 10, 2, pp. 239-259.

DOWLING R. (2008), “Geographies of identity : labouring in the 'neoliberal' university”, Progress in Human Geography, 32, 6, pp. 812-820.

FUNDACIO MARIA AURELIA CAPMANY (2007), Conciliació i nous usos del temps, Collecció Eines, 8, Barcelona, Institut Català de les Dones.

GARCIA DE CORTAZAR M. \& GARCIA DE LEON M. A. (1997), Mujeres en minoría. Una investigación sociológica sobre las catedráticas de universidades de España, Centro de Investigaciones Sociológicas, col. Opiniones y Actitudes, 16.

GARCIA DE LEON M. A., GARCÍA DE CORTÁZAR, M. \& ALONSO M.J. (2001), Las académicas. Profesorado universitario y género, Madrid, Instituto de la Mujer, 72.

GARCIA RAMON M.D., CASTAÑER M., CLOS I. (1988), “Women and Geography in Spanish Universities”, The Professional Geographer, 40, 3, pp. 307-345.

GARCIA RAMON M.D., NOGUÉ, J. \& ALBET A. (1992), La práctica de la geografía en España (1940-1990), Innovación metodológica y trayectorias individuales en la geografía académica española, Barcelona, Oikos-tau (Colección "Estudios Geográficos").

GARCIA RAMON M.D. \& PUJOL H. (2004), “Gender representation in academic geography in Catalonia (Spain)", Journal of Geography in Higher Education, 28, 1, pp. 111-119.

HANSON S. (2000), “Networking”, The professional Geographer, 4, 52, pp. 751-758.

HANSON S. (2007), "Service as subversive activity : on the centrality of service to an academic career", Gender, Place and Culture, 14, 1, pp. 29-34.

KNIGHTS D. \& RICHARDS W. (2003), "Sex discriminations in UK academia", Gender, Work and Organization, 10, 2, pp. 213-238.

KREFTING L.A. (2003), "Interwined Discourses of Merit and Gender : Evidence from Academic Employement in the USA", Gender, Work and Organization, 10, 2, pp. 260-278.

MARTINEZ COSTA C. (2008), El acceso a la cátedra en las Politécnicas, Mujeres en la alta dirección. La carrera profesional de las mujeres en la empresa, la administración y la universidad, Madrid, Instituto de la Mujer.

McDOWELL L. (1979), “Women in British Geography”, Area, 2, 11, pp. 151-154.

MIYARES A. (2008), De catedráticas a rectoras ¿Dónde están las mujeres ?, Mujeres en la alta dirección. La carrera profesional de las mujeres en la empresa, la administración y la universidad, Madrid, Instituto de la Mujer.

MONK J. (1994), “Place Matters : Comparative International Perspectives on Feminist Geography", The Professional Geographer, 46, 3, pp. 277-288.

MOMSEN J. (1980), “Women in Canadian Geography”, The Profesional Geographer, 32, 3, pp. 365-369. PEREZ SEDEÑO E. (coord.) (2008), La situación de las mujeres en el sistema educativo de ciencia y tecnología en España y su contexto internacional, Programa de análisis y estudios de acciones destinadas a la mejora de la calidad de la enseñanza superior y de actividades del profesorado universitario (REF : S2/ EA2003-0031), 207 pp. , http://www.usc.es/IMG/pdf/EA2003-0031.pdf

PUJOL H. (2004), “De la geògrafa absent a la geògrafa desapareguda ?”, Documents d'Anàlisis Geogràfica, 45, pp. 135-142. 
PUJOL H. (2011), La presència de les dones a la geografia acadèmica : de la geògrafa absent a la geògrafa desapareguda, Bellaterra, Universitat Autònoma de Barcelona, thèse de doctorat non publiée.

PUJOL H., ORTIZ A. \& GARCIA RAMON M.D. (2008), “La presencia y la carrera profesional de las mujeres en la geografía académica, Estudios Socioterritoriales", Revista de Geografía, VII, 7, pp. 136-159.

RAMIRO E. (2005), "Discriminació de la dona a les revistes de geografia valencianes. Estudi bibliomètric", Treballs de la Societat Catalana de Geografia, 59, pp. 131-155.

SIMELIO N. \& ROVETTO F. (2008), "La mujer joven en la universidad ¿Una doble discriminación ?", Actas del I Congreso Internacional sobre sesgo de género y desigualdades en la evaluación de la calidad académica, Bellaterra, Universitat Autònoma de Barcelona, pp. 21-33.

TOBIO C. (2005), Madres que trabajan. Dilemas y estrategias, Madrid, Ediciones Càtedra.

TOMAS M., DURAN M., GUILLAMÓN C. (2008), La implicación de las profesoras en la gestión universitaria. Actas del I Congreso Internacional sobre sesgo de género y desigualdades en la evaluación de la calidad académica, Bellaterra, Universitat Autònoma de Barcelona, pp. 195-205.

UNIVERSITAT AUTONOMA DE BARCELONA (2008), Primer Pla d'Acció per a la igualtat entre dones $i$ homes a la UAB, 2006, http://publicacions.uab.es/cd/tot/cat/pla_igualtat_homes_i_dones.pdf.

WILSON F. \& NUTLEY S. (2003), "A critical Look at Staff Appraisal : The Case of Women in Scottish Universities”, Gender, Work and Organization, 10, 3, pp. 301-319.

WINKLER J. A. (2000), “Faculty reappointment, Tenure, and Promotion ; Barriers for Women”, The Professional Geographer, 4, 52, pp. 737-750.

WOOD G.J. \& NEWTON J. (2006), “Childlessness and Women Managers : ‘Choice', Context and Discourses" Gender, Work and Organization, 13, 4, pp. 338-358.

ZELINSKY W (1973a), “The strange Case of the Missing Female Geographer”, The Professional Geographer, 2, 25, pp. 101-105.

ZELINSKY, W. (1973b), "Women in Geography : a brief factual account", The Professional Geographer, 2, 25, pp. 151-165.

\section{NOTES}

1. Cet article s'inscit dans une recherche financé par IEC (PT2008-50501-GCIARAMON01), AGAUR (SGR-2009-1321) et MICIIN (CS0 2009-10913).

\section{RÉSUMÉS}

Des processus de néo-libéralisation s'installent au sein du monde universitaire avec la présence grandissante de logiques compétitives et de marché, de procédures d'audits et des requis de responsabilité/imputabilité et avec le remplacement de fonds publics par des fonds privés. Ces processus ont un impact important sur les perceptions et pratiques relatives à la carrière professionnelle des femmes géographes au sein des départements espagnols de géographie, un 
espace de plus en plus compétitif et masculin. L'article prête une attention particulière aux stratégies et pratiques des femmes en ce qui concerne la conciliation vie professionnelle/vie familiale, ainsi qu'à l'accès aux promotions et au pouvoir. Néanmoins, nous nous intéressons aussi au vécu des hommes dans le monde académique pour ébaucher une comparaison entre les carrières professionnelles masculines et féminines. La recherche est basée sur l'analyse qualitative, notamment des interviews en profondeur.

The ongoing processes of neoliberalisation in universities entail an increase of competition based on the logic of markets, the introduction of managerial procedures of assessment and requirements of accountability alongside with the substitution of private for public funding. These processes have a deep impact on the perceptions and practices related to the professional career of women geographers in Geography departments in Spain, an increasingly competitive and masculine space. The article pays particular attention to the strategies and practices of women with regard to the conciliation of professional and family life as well as to their promotion and to access to power positions. Attention is also paid to the experience of men in the same context in order to sketch a comparison of men and women's careers. The research is based on a qualitative analysis mainly of in-depth interviews.

\section{INDEX}

Keywords : gender, university, geography, Spain

Mots-clés : genre, université, géographie, Espagne

\section{AUTEURS}

\section{MARIA DOLORS GARCIA RAMON}

Département de Géographie, Universitat Autònoma de Barcelona, mariadolors.garcia.ramon@uab.es

\section{ANNA ORTIZ}

Département de Géographie, Universitat Autònoma de Barcelona, anna.ortiz@uab.es

HERMINIA PUJOL

Centre d'Estudis Demogràfics, CED, Universitat Autònoma de Barcelona, hpujol@ced.uab.es 